\title{
Contribution of Prof. Dr. Mahendra Pal in Cutaneous Mycoses
}

\section{Pall $\mathbf{M}^{*}$ \\ Director of Veterinary Public Health and Microbiology, India}

*Corresponding author: Mahendra Pal, Founder Director of Narayan Consultancy on Veterinary Public Health and Microbiology, Gujarat, India, Email: palmahendra2@gmail.com
Short Communication

Volume 3 Issue 2

Received Date: August 03, 2020

Published Date: August 13, 2020

DOI: $10.23880 /$ oajmms-16000125

\section{Short Communication}

Fungi are a diverse group of heterotrophic, eukaryotic, achlorophyllous, Gram positive, non-acid fast, living organisms that multiply sexually and asexually, and exist in our environment as evidenced by their isolation from the soil, air, water, grains, fruits, vegetables, woods, avian droppings, etc [1]. Fungi have the potential to cause disease in plants, animals, and humans. Fungal infections are observed in both sexes, in all age groups, in all seasons, in rural and urban settings, in all climatic zones, and in every strata of society; and are important from public health as well as economic point of view. There are around 5 million species of fungi in the universe, of which only 600 have been found to be concerned with infections in humans and animals including birds [2]. The diseases of warm blooded animals caused by various fungi, is called mycoses, which are global in distribution. The dramatic rise in the immunocompromised population has resulted an increased incidence and prevalence of fungal infections, and thus presenting a gigantic challenge to the health care professionals. The mycotic infections are recognized as significant cause of morbidity and mortality in humans as well as in animals [1]. The cutaneous mycoses are the fungal infections caused by dermatophytes, yeasts, and non-dermatophytic moulds, and are reported in both sexes, in all age groups, in all seasons, in rural and urban settings, and from developing as well as developed nations of the world $[1,3]$. The cutaneous fungal infections are affecting more than $20-25 \%$ of the world's population $[3,4]$. Dermatophyosis is the most commonly reported cutaneous mycosis in medical and veterinary clinics throughout the world. The disease is mostly occurs in sporadic form but outbreaks are also reported, and is still considered as a major public health problem in many regions of the world [5-7]. Dermatophytes are the filamentous fungi that have the potential to affect the skin, nail and hair [1]. It has three genera, namely, Epidermophyton, Microsporum and Trichophyton, and all of which can cause cutaneous mycosis [1]. A number of factors that include hygiene, life style, age, host status, climate, socioeconomic conditions, and presence of animals particularly the pets govern the prevalence of dermatophytosis [1]. The causative significance of yeasts (Candida, Cryptococcus) and non-dermatophytic moulds (Alternaria, Aspergillus, Fusarium, Geotrichum, Scopulariopsis and others ) in cutaneous mycoses is also documented by several researchers including the author Pal M, et al. [3,8-12].

Prof. Dr. Mahendra Pal is a globally recognized scientist who had opportunities to work in Veterinary and Medical Colleges in India and abroad. He has published over 655 papers, 8 books and several awards/prizes to his credit. Out of 655 publications, over 65 papers are related to cutaneous mycoses of humans and animals. Prof. Pal has published papers on numerous mycotic diseases including aspergillois, candidiasis, coccidiodomycosis, cryptococcosis, dermatophyosis, fusariosis, geotrichosis, histoplasmosis, mycetoma, sporotrichosis, rhinosporidiosis, and zygomycosis $[1,13]$. His book entitled "Veterinary and Medical Mycology", the First in India, is very much valued by the scientists, academicians, researchers, teachers, and students globally. Prof. Pal guided/advised over 67 students at DVM, MSc, MVSc, and Ph.D. level in India as well as in Ethiopia [13]. He has established for the first Time the prevalence of Cryptococcus neoformans in the environment of New Zealand, Nepal, and Djibouti. Prof. Pal has the credit to develop sunflower seed medium (Pal medium), APRM (Anubha, Pratibha, Raj, Mahendra) medium, PHOL (Pal Hasegawa, Ono, Lee) stain, and Narayan stain for the studies of fungi that are implicated in various clinical disorders of humans and animals $[1,12,14-$ 16]. He is credited to elucidate for the first time the etiologic significance of Tricochosporon beigelii in burn wound of cattle [17], Trichophyton verrucosum in dermatitis of barking deer [6], Exserohium rostratum in equine dermatitis [18], Aphanoascus fulvescens in canine dermatitis [19], Candida tropicalis in dermatitis of rabbit [20], and Aspergillus terreus in buffaloes [9]. Prof. Pal has also described dermatophytosis 


\section{Open Access Journal of Mycology \& Mycological Sciences}

in monkey, hog deer, bear, lion, barking deer for the first time in India [1]. He has investigated one severe outbreak of dermatophytosis in cattle in 1987 from India Pal M, et al. [21], and a small outbreak of ringworm in barking deer in 1993 from Nepal [6]. The first mycological investigation of dermatophytosis in camels due to Trichophyton verrucosum by conducted by Pal in Ethiopia [22].

His comprehensive and systematic studies in the field of veterinary and medical mycology during last five decades revealed that family pets can serve as a source of Microsporum canis, an emerging zoophilic dermatophyte of global significance [14]. Prof. Pal reported that Trichophytn rubrum, an important anthropophilic dermatophyte, can be transmitted from humans to animals, such as cattle, dog, goat, and monkey [1,23]. Further, it is important to mention that human can get zoophilic dermatophytes from several animals including dog, cat, cattle, buffalo, goat, horse, monkey, and camel [1,5,7,24-32]. An interesting case of chronic tinea pedis infection due to two dermatophtyes, namely Microsporum canis and Trichphyton rubrum was described for the first time in India Pal M, et al [33]. Cutaneous mycosis due to Cryptococcus neoformans in a parrot handler Pal M, et al. [11] and Aspergillus terreus in an agricultural worker Dave $\mathrm{P}$, et al. [12] constituted the first report from Western India.

Recently, Prof. Pal founded "Narayan Consultancy on Mycology" to offer free technical advice to the scientists, researchers, and students particularly belonging to poor resource countries of the world. Based on his immense contribution in animal mycoses, Prof. Pal is considered as "Father of Veterinary Mycology" in India [34,35]. His 520 papers uploaded on international websites are viewed by more than 370,000 scientists of 171 countries of the world. Currently, more than 2000 students are benefited by the knowledge and wisdom of Prof. Pal. His papers are frequently cited by many scientists, researchers and academicians in their papers, reviews, books, and monographs. Prof. Pal delivered several guest lectures on mycotic diseases of humans and animals in medical and veterinary faculties in New Zealand, Belgium, Japan, Ethiopia, and India, and also helped few medical colleges in India to develop medical mycology.

It is emphasized that comprehensive and systematic studies should be conducted to establish the etiologic role of yeasts and non-dermatophytic moulds in cutaneous mycoses. Further, the efficacy of Pal sunflower seed medium for the isolation of yeasts and non-dermatophytic moulds; and Narayan stain to undertake detailed morphological studies of fungi isolated from cutaneous lesions is suggested. There is a need to develop safe, potent, and low cost therapeutic agents that can be easily affordable by the poor resource countries to mitigate the morbidity and mortality due to most frequently encountered fungal diseases. As mycotic diseases are still neglected as compared to other infectious diseases, it seems imperative to provide sufficient funding to conduct detailed research on various aspects of mycoses that have a great impact on the health of humans and many species of animals.

\section{References}

1. Pal M (2007) Veterinary and Medical Mycology. $1^{\text {st }}$ (Edn.), Indian Council of Agricultural Research Institute, New Delhi, India.

2. Pal M (2018) Emerging role of saprobic fungi in human and animal health. J Mycopathol Res 56(3): 1-2.

3. Pal M, Kerorsa GB (2020) Growing significance of nondermatophytic fungi in cutaneous disorders of humans and animals. Journal of Microbiology, Immunology, and Biotechnology 7: 27-32.

4. Havlickova B, Czaika VA, Friedrich M (2008) Epidemiological trends in skin mycoses worldwide. Mycoses 51(4): 2-15.

5. Pal M (1991) Tinea barbae in an animal attendant due to Trichophyton mentagrophytes. Livestock Advisor 16: 21-23.

6. Pal M, Thapa BR (1993) An outbreak of dermatophytosis in barking deer (Muntiacus muntjak). Vet Record 133(14): 137-348.

7. Pal M (2001) Dermatitis in a goat and its handler due to Microsporum canis. J Anim Sci 1: 138-139.

8. Pal M, Mehrotra BS (1983) Occurrence and etiologic significance of Cryptococcus neoformans in a cutaneous lesion of a cat. Mykosen 26(12): 608-610.

9. Pal M (2003) Role of Aspergillus terreus in dermatitis of buffaloes. Intas Polivet 4: 314-316.

10. Vyas A, Pathan N, Sharma R, Vyas L (2013) A Clinicomycological study of cutaneous mycoses in Sawai Man Singh Hospital of Jaipur, North India. Ann Med Health Sci Res 3(4): 593-597.

11. Dave P, Pal M (2015) Primary cutaneous mycosis in an immunocompetent parrot keeper due to Cryptococcus neoformans. Molecular Microbiology Research 5(4): 1-3.

12. Dave P, Mahendra R, Pal M (2015) Etiologic significance of Aspergillus terreus in primary cutaneous mycosis of an agricultural worker. Molecular Microbiology Research 5(2): 1-4. 


\section{Open Access Journal of Mycology \& Mycological Sciences}

13. Pal M (2019) Contribution of Prof. Dr. Mahendra Pal in Veterinary and Medical Mycology. Open Acess Journal of Mycology and Mycological Sciences 2(2): 1-2.

14. Pal M, Hasegawa A, Ono K, Lee CW (1990) A new staining solution for the; morphological studies of fungi and Prototheca. Nihon Juigaku Zasshi 52(3): 527-531.

15. Pal M (1997) Use of Pal's sunflower seed medium for any early diagnosis of cryptococcosis. The Antiseptic 95(5): 175.

16. Dave P, Pal M (2015) New medium "APRM" for isolation of medically important fungi from clinical and environmental samples. Int J Livest Res 5(3): 10-18.

17. Pal M, Salkin F, Dixon DM (1991) Colonization of a bovine wound by Trichosporon beigelii. Mycoses 34(11-12): 513-514.

18. Pal M, Lee CW (1994) Exserohilum rostratum: First isolation from equine dermatitis. Korean Journal of Veterinary Clinical Medicine 11: 187-189.

19. Pal M (1995) Aphanoascus fulvescens: First report of its isolation from canine dermatitis in India. Rev Iberoam Micol 12: 1.

20. Pal M, Rao MN (1999) Dermatitis in a rabbit caused by Candida tropicalis. Livestock International 3: 10-11.

21. Pal M (1987) Dermatophytosis in cattle: clinical and mycological studies. Indian Journal of Animal Sciences 57: 856-858.

22. Pal M (2016) First mycological investigation of dermatophytosis in camels due to Trichophyton verrucosum in Ethiopia. Journal of Mycopathological Resarch 54(1): 89-92.

23. Pal M, Matsusaka N, Chauhan P (1997) Zooanthroponotic significance of Trichophyton rubrum in a rhesus monkey (Macana mulatta). Verh Ber Erkrg Zootier 38: 355-358.

24. Pal M, Matsusaka N (1991) Dermatophytosis in a rhesus monkey (Maccaca mulatta) and animal attendant caused by Microsporum canis. Verh Ber Erkrg Zootier 33: 271 264.

25. Pal M (1997) Dermatophytosis in a bear due Microsporum gypseum. Zoos' Print 12: 13-14.

26. Pal M, Lee CW (1997) Microsporum canis infection in a horse and its transmission to man. Korean Journal of Veterinary Clinical Medicine 15: 196-198.

27. Pal M (1988) Ringworm in a lion (Panthera leo) caused by Microsporum gypseum. Wildlife Health 6: 1-4.

28. Pal M, Lee CW (2000) Trichophyton verrucosum infection in a camel and its handler. Korean Journal of veterinary Clinical Medicine 17: 293-294.

29. Pal M, Dave P (2006) Transmission of Trichophyton verrucosum infection from cattle to man. Intas Polivet 7: 429-431.

30. Dave P, Mahendra R, Pal M (2014) Growing significance of Microsporum canis in tinea of animal handlers. J Environ Occup Health 3(4): 193-195.

31. Dave P, Pal M (2015) Tinea manum in a veterinarian caused by Trichophyton verruvosum. Ethiopian International Journal of Multidisciplinary Research 2(4): 10-12.

32. Dave P, Pal M (2017) Ringworm in a butcher caused by Trichophyton verrucosum. Indian Journal of Veterinary Public Health 3(2): 33-35.

33. Pal M, Chauhan P (1994) Chronic tinea pedis due to Microsporum canis and Trichophyton rubrum. Rev Iberoam Micol 11: 5- 6.

34. Pal M, Lee CW, Matsusaka N (2002) Occurrence of Geotrichum candidum in two cases of canine dermatitis. Journal of Veterinary Clinics 19(4): 433-435.

35. Pal M (2004) Efficacy of Narayan stain for morphological studies of moulds, yeasts and algae. Rev Iberoam Micol 21(4): 219.

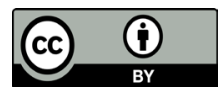

